\title{
LE CONDIZIONI DI SOSTENIBILITÀ PER L'ATTIVITÀ DI TUTELA
}

\author{
PAOLA DUBINI $(*)$
}

SUNTO. - L'articolo esamina la specificità dell'attività di tutela da un punto di vista economico e gestionale e le sue condizioni di sostenibilità. Pur considerando la centralità della tutela nelle politiche culturali e l'indispensabile ruolo pubblico nel suo esercizio, l'articolo suggerisce la necessità che diverse categorie di stakeholder siano coinvolti nella consapevolezza della sua rilevanza e nel suo sostegno.

$* * *$

ABSTRACT. - The article examines heritage conservation from an economic viewpoint and considers the conditions for its sustainability. Conservation is crucial in any cultural policy, and the governmental role is fundamental in its support. However, the article suggests a much broader involvement of different stakeholders, both in acknowledging its relevance and in supporting it.

In questo intervento mi propongo di riflettere sulle condizioni di sostenibilità per l'attività di tutela ${ }^{1}$. In estrema sintesi, il mio ragionamento si snoda attorno ai seguenti punti.

L'attività di tutela è centrale in ogni politica culturale legata al patrimonio; la tutela origina ricerca, conoscenza, possibilità di valorizzazione, una fruizione potenzialmente più consapevole e quindi un beneficio collettivo, che si riverbera positivamente sull'attività di tutela, contribuendo a renderla più efficace ed efficiente; un ragazzino che non

(*) Università L. Bocconi di Milano, Italia. E-mail: paola.dubini@unibocconi.it

1 Desidero ringraziare il centro ASK dell'Università L. Bocconi di Milano per il sostegno ricevuto nella stesura del presente articolo. 
prende a pallonate una fontana barocca perché sa che è fragile e preziosa contribuisce a ridurre il costo della tutela.

$\mathrm{Da}$ un punto di vista economico, tuttavia, la tutela è un investimento il cui valore e ritorno non sono percepiti facilmente; inoltre, gli investimenti e i costi associati all'attività di tutela sono molto anticipati rispetto ai possibili risultati positivi generati. Non è quindi scontato che l'importanza della tutela sia riconosciuta a priori. Diventa allora necessario che tale attività sia "militante", nel senso di essere capace di affermare con forza la sua importanza e di coinvolgere attori di natura diversa e di non essere "confinata" da un punto di vista organizzativo.

Perché ciò avvenga in modo efficace ed efficiente, è utile che sia progettata e pensata collegata al processo di valorizzazione e che diventi responsabilità collettiva; questo non è un passaggio facile, perchè richiede cambiamenti sia all'interno dell'organizzazione statale, sia nei rapporti con l'esterno. La riforma del MIBACT in atto ha attivato alcuni processi in questa direzione.

Alcuni esperimenti di crowdsourcing in ambito culturale, con particolare riferimento alle biblioteche, aprono interessanti prospettive di riflessione su come realizzare una attività di tutela sostenibile, valorizzando al contempo la dimensione materiale e immateriale del patrimonio.

Ciascuno dei passaggi evidenzia non pochi aspetti problematici, trattati in modo puntuale da una letteratura specializzata e lontani dall'essere risolti. Non ho modo in questo articolo di affrontarli con la necessaria profondità e con sufficiente rigore; preferisco quindi concentrarmi sul ragionamento nel suo complesso e in particolare sul contributo che una lettura di taglio economico in una prospettiva di sostenibilità può dare alla causa della conservazione e della tutela del nostro patrimonio.

\section{LA CENTRALITÀ DELL'ATTIVITÀ DI TUTELA}

E' noto che la politica culturale nel nostro paese ha tradizionalmente messo al centro l'attività di tutela e che sul tema l'Italia è riconosciuta a livello internazionale; l'articolo 9 della Costituzione, il comando dei carabinieri per la tutela del patrimonio culturale, la task force italiana dell'UNESCO per l'intervento nelle aree di crisi, la riconosciuta competenza dell'Italia in materia di restauro, dal punto di vista teorico e applicativo, il lavoro delle sovrintendenze sui territori sono fra le espressioni - 
conosciute anche a un pubblico di non specialisti - della attenzione istituzionale alla tutela ed evidenze di una tradizione di conservazione consolidata. Il dettato costituzionale parla di "responsabilità della Repubblica" a tutelare e l'azione collettiva deve essere orientata a proteggere e conservare per le prossime generazioni, creando le premesse per una responsabile attività di valorizzazione, cosicché effettivamente il patrimonio sia rappresentativo della nostra identità e si mantenga vivo nella nostra attenzione, nel nostro affetto e nel nostro sostegno.

E' altrettanto vero però che - riguardo alla tutela - negli anni sono via via emersi numerosi aspetti problematici, analizzati da Cecchi (2015), che hanno alimentato un intenso dibattito (Manacorda 2014). Il fronte della riflessione è ampio e articolato; già il tema su "che cosa" sia patrimonio - e quindi su "che cosa" debba orientarsi l'attività di tutela - è aperto (Chastel in Emiliani 1980; Dezzi Bardeschi 2004; Piergigli 2006). Non sappiamo quanto sia grande il patrimonio, né come attribuirgli un valore, né quanti tipi di valore attribuirgli e quindi quali indicatori utilizzare per misurarli; di sicuro il patrimonio è "tanto", in costante aumento, e nonostante l'attenzione al tema e gli sforzi profusi, l'efficacia della tutela è ampiamente migliorabile.

Inoltre, l'enfasi sui beni culturali rispetto alle aziende che ne hanno responsabilità di conservazione e gestione ha portato a mettere sullo stesso piano realtà molto diverse fra loro (ad esempio i musei, gli archivi e le biblioteche e il paesaggio) (Casini 2016) e la dimensione del built heritage rispetto al patrimonio immateriale; questo spiega fra l'altro perché, nonostante il nostro paese sia fra i più attivi sostenitori dell'UNESCO e riconosciuto nel campo della conservazione, non abbia ancora ratificato la convenzione quadro del Consiglio d'Europa sul valore del patrimonio culturale per la società (cd Convenzione di Faro), a causa di un mancato allineamento del nostro impianto normativo alla definizione internazionale di patrimonio, che incorpora la componente immateriale. Sul primo aspetto, la riforma Franceschini del 2014 rappresenta un cambiamento significativo all'impianto normativo nazionale, giacché individua attori specifici, i musei e i poli, responsabilizzati sulla valorizzazione, permettendo alle Sovrintendenze di concentrarsi sull'attività di tutela.

Ancora, l'attività di tutela deve considerare la dimensione sempre più globale delle arti e del patrimonio; questo si traduce in una maggiore attenzione al patrimonio e in una richiesta crescente di prestiti e di movimentazione di opere, ma anche purtroppo in una estensione delle 
azioni di violazione e commercio illegale (Casini 2010), mettendo sotto pressione gli organi responsabili dell'azione di tutela.

Il Codice dei Beni Culturali (Sciullo in Cammelli 2004) affida compiti precisi al MIBACT in materia di tutela: riconoscimento, individuazione, protezione e conservazione. La dimensione, la stratificazione e talvolta la pesantezza dell'apparato normativo fanno sì che l'applicazione del dettato di legge possa essere molto diversa in diversi contesti (Petraroia; 48 in Montella e Dragoni 2010), limitando in modo significativo l'efficacia della tutela e non contribuendo alla sua buona nomea agli occhi dell'opinione pubblica. In assenza di parità di trattamento, in presenza di opacità informativa, difficilmente il cittadino medio guarda alla sovrintendenza con gratitudine per il lavoro svolto nel proteggere un patrimonio meraviglioso, di tutti, di cui andare orgogliosi e da cui imparare.

Per quanto il dibattito sulla importanza della tutela e sulla efficacia dell'azione di governo nella trasformazione del MIBACT sia articolato e con posizioni molto diverse fra loro, molti concordano sulla forte limitatezza delle risorse - umane e finanziarie - destinate alla tutela (Settis 2005; Montanari 2014; Cecchi 2015 per citare alcuni contributi recenti) e sulla scarsa possibilità di aggiornamento e rinnovamento professionale rispetto alla dimensione del patrimonio e dei crescenti e mutevoli fabbisogni di tutela e di valorizzazione. La riduzione delle risorse pubbliche destinate in generale alla gestione del patrimonio nel periodo 2008-2015 (i risultati più recenti sono per fortuna in controtendenza per quanto riguarda gli investimenti statali), e quindi anche alla sua tutela, è oggetto di allarme e di preoccupazione diffusi; ciò è particolarmente vero in questo momento, in cui il clima di incertezza a livello internazionale e la crescita nelle minacce di terrorismo da una parte e crolli a seguito delle ripetute scosse di terremoto dall'altra hanno mostrato quanto il nostro patrimonio sia vulnerabile. D'altro canto, sarebbe semplicistico legare la capacità di tutela alle sole dimensioni del personale dedicato; come ricorda Manacorda (2014; 34-35), già la commissione Franceschini aveva tratto "sicuro convincimento" che "lo stato di precarietà e decadenza del patrimonio (...) non può essere attribuito esclusivamente, e neppure prevalentemente, ad una deficienza quantitativa di personale e di finanziamento delle competenti amministrazioni pubbliche di tutela" e, in tempi più recenti, Urbani (2000) aveva rilevato un aumento di organico significativo all'interno delle Soprintendenze e del Ministero, non accompagnato da un miglioramento nella qualità dei risultati ottenuti.

Come è possibile che una attività che comunque rimane fonda- 
mentale nelle politiche culturali del nostro paese possa non essere adeguatamente riconosciuta e quindi anche finanziata? Al di là di considerazioni di natura politica, la teoria economica evidenzia come elemento intrinseco di quest'attività la sua natura "meritoria". I beni meritori (Musgrave 1959) hanno due caratteristiche principali: innanzitutto, a differenza dei beni privati, il cittadino non sa o non è in grado di valutare quale sarà il suo beneficio diretto derivante dal fatto che un attore economico (sia esso pubblico o privato) svolga questa attività e ne sostenga i relativi costi. Inoltre, l'attività di tutela genera un beneficio alla collettività (la letteratura economica lo chiama esternalità positiva) da cui la collettività trae guadagno (economico o non economico), ma è difficile mobilitare la collettività a contribuire all'attività di tutela.

Queste due caratteristiche determinano alcune conseguenze rilevanti: la prima è che ci rendiamo conto dell'importanza e del valore della tutela quando il patrimonio è distrutto, da eventi naturali o deliberati. Il pensiero corre inevitabilmente ai danni irreparabili al patrimonio provocati dai terremoti dell'estate scorsa, alle critiche sull'operato del governo, alle promesse di ricostruzione e allo sgomento che hanno suscitato per la vastità del compito. E per quanto riguarda la situazione internazionale, fa riflettere la difficoltà di un organismo come l'UNESCO a salvaguardare il patrimonio, e d'altra parte l'importanza politica del riconoscimento, finalmente, che i crimini contro il patrimonio sono crimini di guerra ${ }^{2}$ (Bevan 2006, Slade 2015). Un'altra conseguenza è che è necessario uno sforzo capillare di informazione (direi quasi di proselitismo) sull'importanza della tutela, perché come tutti i beni meritori è "data per scontata" e soffre di un endemico fallimento informativo; questa attività informativa deve essere tra l'altro orientata a responsabilizzare la collettività sulle ricadute positive dell'investimento in tutela. Poiché è attività i cui risultati si danno per scontati, la tutela è a rischio più di altre di essere oggetto di "tagli che non indignano". Inoltre, è attività che la collettività non fa fatica ad affidare ad altri. Ciascun cittadino avrebbe interesse indi-

2 Mi riferisco qui alla condanna nel 2016 da parte della Corte di Giustizia Internazionale dell'Aia di Ahmed al-Faqi al-Mahdi a nove anni di prigione per la distruzione di nove mausolei e dell'ingresso di una moschea nella città di Timbuktu per suo ordine nel 2012. Si tratta del primo caso di condanna per crimini contro il patrimonio culturale mondiale. 
viduale a partecipare alla tutela del patrimonio: ogni atto di vandalismo o di incuria non porta beneficio individuale, ma in compenso determina costi che ricadono sulla collettività e quindi anche sugli individui. Detta in altro modo, l'investimento individuale nella tutela è piccolo a fronte di un elevato beneficio individuale per le conseguenze dell'attività di tutela, ma la percezione del beneficio e del costo è fortemente falsata, richiedendo un investimento significativo di "educazione del mercato".

Si noti che le attività meritorie possono essere considerate a livello di sistema, ma anche di singola organizzazione. Come ben evidenzia Jalla (2015) con riferimento alla sicurezza dei musei, ad esempio, si tratta di attività del cui valore ci rendiamo conto quando qualcosa va storto, sulle quali siamo portati a non investire quando va tutto bene e che quindi suggeriscono una allocazione piccola (perché c'è poca propensione ad investire su qualcosa di cui fatichiamo a percepire il valore), ma costante nel tempo.

La riflessione sui beni meritori è utilizzata nella teoria economica per spiegare la necessità di un intervento pubblico; poiché il mercato dei beni meritori è imperfetto, per quanto vi possano essere privati disposti ad investire in un bene o in una attività meritoria, l'offerta totale non è sufficiente a garantire un livello sociale efficiente di consumo. Così come molte persone non riconoscono il beneficio per se stessi e per gli altri di fare visite regolari dall'oculista o di vaccinare i propri figli, così, lasciata al mercato, l'attività di tutela sarebbe insufficiente e quindi - come la cronaca ci dice riguardo alle vaccinazioni - inefficace. Ancora, come nel caso del terremoto, l'importanza e il valore di un bene meritorio (e quindi la giustificazione per avere investito o l'accusa di non avere investito abbastanza) spesso emerge quando è troppo tardi, o comunque molto tempo dopo che l'investimento in tutela è stato realizzato.

\section{PER UNA TUTELA SOSTENIBILE}

Il richiamo alla teoria dei beni meritori può a questo punto apparire fuori luogo: dopotutto nessuno mette in discussione il fatto che l'investimento in tutela debba essere realizzato con risorse pubbliche. Il richiamo alla teoria, tuttavia, mi pare rilevante, perché gli stessi motivi che spiegano la scarsa attenzione da parte del mercato (e quindi la scar- 
sa disponibilità ad investire da parte di investitori privati) all'attività di tutela ci aiutano a comprendere, da un punto di vista economico, la difficoltà a garantire risorse pubbliche all'attività di tutela e la necessità di un comportamento propositivo e orientato ai beneficiari, oltre che al patrimonio. Il fatto di riconoscere che un bene è meritorio e quindi necessita di sostegno pubblico perché il mercato privato non è sufficientemente grande e non funziona in modo efficiente per garantire un sufficiente beneficio sociale non garantisce automaticamente che l'attore pubblico sarà disposto a sostenerlo adeguatamente. All'interno della discussione sull'adeguatezza e sull'efficacia dell'investimento in tutela si possono cogliere almeno tre ordini di considerazioni, che in ultima istanza pongono l'accento sui livelli di efficacia e di efficienza nell'uso di risorse pubbliche. In primis, occorre considerare valutazioni di opportunità e di scelta fra alternative; data una quantità di risorse pubbliche, sono diverse le possibilità di investimento a beneficio della collettività. Perché investire in tutela quando altri beni meritori (dall'istruzione alla salute, per citare i più evidenti) richiedono attenzione e risorse e sono per molti più immediatamente collegabili a benefici per la collettività?

Un'altra categoria di freni all'investimento pubblico in tutela è di carattere istituzionale e organizzativo e riguarda l'efficacia e l'efficienza delle strutture preposte all'attività di tutela nella capacità di anticipare i problemi, accelerare i tempi di risposta e proporre soluzioni regolative e conservative e una informativa sempre più efficaci. All'interno di questo dibattito, la riflessione sulla manutenzione programmata è forse quella più ricca e articolata, per le implicazioni istituzionali, gestionali e di rapporti fra enti in termini di capacità dell'attività di tutela di essere efficace ed efficiente nel lungo periodo (Cecchi 2006, Della Torre Petraroia 2008; Della Torre 2010; Gasparoli 2012).

Inoltre, la riforma Franceschini del 2014 ha aperto al riguardo una serie di considerazioni sui nuovi ruoli che strutture diverse all'interno del Ministero devono svolgere per garantirne l'efficacia di funzionamento (Jalla 2015; Forte 2015). Buona parte delle posizioni critiche riguardo alla riforma (si veda fra tutti la posizione di Settis) e in generale al comportamento dello Stato sono relative all'attività di tutela, hanno ad oggetto la riduzione dell'ambito di attività delle Soprintendenze e il loro accorpamento e in generale un "abbassamento della guardia" sulla criticità della tutela.

In una prospettiva economico aziendale, la possibilità di realiz- 
zare una attività di tutela efficace dipende da condizioni istituzionali, condizioni di governo e organizzative (un assetto che faciliti lo svolgimento dell'attività di tutela), la disponibilità di competenze specifiche (di programmazione, di prevenzione, di controllo, di enforcement), una adeguata configurazione dei processi e adeguate risorse economico finanziarie (Montella 2009). Se l'investimento pubblico in tutela non è scontato, è evidente che un miglioramento dell'efficienza nell'uso delle risorse disponibili è condizione necessaria, ma non sufficiente; occorre riflettere sui modi per far sì che la centralità della tutela all'interno delle politiche culturali rimanga sostanziale e che attorno ad essa si raccolga il necessario livello di consenso, premessa indispensabile per raccogliere un maggior numero di risorse e stimolare l'attrazione e la valorizzazione delle tante competenze in cui la tutela si declina.

Il dibattito recente in questa direzione può essere così sintetizzato (Cammelli, Covatta 2013 in Manacorda 2014;22): "Il rinnovamento della "cultura della tutela" passa attraverso quattro prese di posizione: riguardo all'oggetto, che non deve essere costituito dalle "cose" ma dai contesti in cui queste cose insistono; riguardo al soggetto, che non deve essere più solo "lo Stato", ma anche la "società civile", riguardo alla procedura, che non deve ridursi al "vincolo amministrativo" ma anche a quello che potrebbe definirsi "vincolo civico", riguardo agli strumenti, che non devono più ridursi a pratiche puntiformi di "restauro estetico", ma piuttosto alla "manutenzione programmata"."

L'affermazione che la tutela non sia responsabilità solo "dello Stato" ma anche "della società civile" ha diverse conseguenze; ne considero due:

- la partecipazione di un più ampio numero di attori al sostegno e alla sostenibilità della tutela;

- l'importanza di coinvolgere i cittadini (in quanto beneficiari) nell'attività di tutela, in una continuità ideale fra conservazione e valorizzazione.

Riguardo al primo punto, la visione sintetizzata da Cammelli e Covatta riconosce la necessità da una parte e l'evidenza dall'altra che per quanto la responsabilità regolativa, ispettiva e autorizzativa spetti al Ministero - altri attori pubblici e privati sono parte in causa e attori della tutela, in un processo collettivo di riconoscimento di appartenenza, di attribuzione di significato e di valore. Anche l'evoluzione normativa da una parte contrappone l'interesse pubblico alla conservazione 
del bene rispetto al privato proprietario, ma dall'altra riconosce che attorno al patrimonio devono contemperarsi l'interesse pubblico, quello privato proprietario e quello collettivo (Bruno 2011) e propone forme di incentivo dell'azione privata a sostegno dell'attività di tutela (si pensi ad esempio al decreto art bonus). Questa mobilitazione di attori diversi è orientata a garantire da un lato la sostenibilità dell'attività di tutela in sé, ma dall'altro a dimostrare che la tutela è premessa per uno sviluppo collettivo sostenibile (Moreno, Santagata, Tabassum 2004).

Per un attore che opera in ambito culturale (Baia Curioni 2006), la sostenibilità è riconducibile alla capacità di operare con continuità in equilibrio su mercati diversi molto diversi fra loro e non governati solo da scambi di natura monetaria:

- un mercato istituzionale, rappresentato dagli enti della pubblica amministrazione, sul quale il valore e la reputazione si misurano in termini politici;

- un mercato di pari, che legittima e consacra. Da questo punto di vista quanto più l'attività di tutela si collega a quella di ricerca, tanto maggiore sarà il suo valore percepito;

- i mercati dei cittadini/dei pubblici/dei clienti, che si attivano in parte in termini di scambi economici, in parte attraverso una maggiore chiarezza informativa e una più veloce risposta nei processi autorizzativi;

- i mercati delle risorse (finanziarie, umane, tecnologiche...).

Poiché l'attività di tutela impiega risorse economiche ma non ne genera direttamente, è attraverso le interazioni con gli altri mercati che puo' assicurarsi la necessaria attenzione e garantirsi nel tempo le risorse che necessita. Riconoscere quindi che la tutela è "affare di tutti" rende necessario uno sforzo sistematico e pianificato di mobilitazione di diverse categorie di interlocutori istituzionali attorno alla centralità del patrimonio e alla responsabilità collettiva e condivisa della sua tutela; perché ciò accada, occorre non solo sviluppare un progetto culturale forte e convincente, ma simultaneamente mettere in atto meccanismi istituzionali che facilitino la partecipazione e la corresponsabilizzazione, sviluppare una cultura organizzativa del "render conto", mobilitare una parte di risorse nella direzione di un maggiore coinvolgimento dei pubblici, non solo fra chi si occupa di valorizzazione, ma anche fra chi si occupa di tutela. Lo strumento dell'art bonus rappresenta una possibile modalità di coinvolgimento di più attori alle istanze della tutela; incentiva i privati a donare attraverso la leva fiscale, ma stimola anche 
gli enti locali e le singole istituzioni culturali a mobilitarsi per stimolare l'iniziativa privata. Se si osserva la dinamica delle donazioni sul sito di art bonus è possibile intravvedere alcuni esempi di sforzo collettivo ad utilizzare lo strumento per mobilitare una varietà crescente di attori rispetto ai "soliti donors".

E' evidente che l'esercizio di sollecitazione, non semplice, richiede al contempo che gli altri interlocutori siano in grado di cogliere le istanze della tutela. Da questo punto di vista, mi pare che la crescente attenzione allo sviluppo sostenibile possa facilitare la diffusione di una cultura della tutela maggiormente condivisa. Come è noto, a partire dalla seconda metà del secolo scorso si è avviata una riflessione sulla necessità di integrare indicatori macroeconomici consolidati - come il Pil, il tasso di inflazione o il rapporto deficit/Pil - con indicatori che permettessero si incorporare nella misura dello sviluppo istanze di:

- conservazione e protezione del suolo, delle risorse e della qualità dell'aria e dell'acqua, di minimizzazione degli scarti e dei rifiuti, e del riutilizzo (sostenibilità ambientale);

- riduzione delle diseguaglianze, accettazione delle diversità, riduzione della povertà economica morale e sociale, accesso all'istruzione, alla salute e alle opportunità per tutti (sostenibilità sociale);

- utilizzo efficace ed efficiente delle risorse, per generare adeguata ricchezza a remunerare i fattori di produzione, a garantire continuità e autonomia di azione agli attori economici e sociali (sostenibilità economica).

Nel processo di costruzione di indicatori di sviluppo che incorporino elementi economici sociali ed ambientali e siano orientati al lungo periodo, gli istituti nazionali di statistica europei hanno proposto indicatori BES (Benessere Equo e Sostenibile). L'Istat pubblica dal 2013 un rapporto BES e dal 2016 gli indicatori BES sono inclusi tra gli strumenti di programmazione e valutazione della politica economica nazionale, in collegamento con i 17 SDG (Sustainable Development Goals) che le Nazioni Unite hanno adottato come obiettivi di sviluppo 2030. Una delle 12 dimensioni di benessere riguarda il paesaggio e il patrimonio e uno specifico obiettivo fra gli SDG (l'11.4) auspica un rafforzamento degli sforzi per proteggere e conservare il patrimonio mondiale naturale e culturale all'interno di un più ampio obiettivo di rendere le città e le comunità sostenibili. L'elaborazione di misure di sintesi porta inevitabilmente alla luce questioni di fondo e criticità di metodo. E in entrambi i casi la strada che porta al disegno, alla messa in atto e alla valuta- 
zione di politiche e di comportamenti sistematicamente orientati alla sostenibilità in una logica integrata è ancora molto lungo. A titolo di esempio, il rapporto BES 2016 (Istat 2016) si limita a considerare due indicatori per la cultura: il numero di beni e istituti culturali (densamente e capillarmente distribuiti su tutto il territorio italiano) e la spesa pubblica per la gestione del patrimonio (inferiore alla media europea e con forti disparità tra Nord e Mezzogiorno); e anche i dati divulgati dal Ministero riguardo all'efficacia dell'azione di tutela sono estremamente poveri in termini di efficacia informativa. E' evidente quindi che perché questi indicatori siano effettivamente strumenti di politica e abbiano forte valore segnaletico sarà necessario studiare e attendere non poco. Tuttavia, tale riconoscimento è senza dubbio un punto di partenza importante e può essere l'occasione per orientare le priorità delle decisioni politiche, avvicinandole a una prospettiva che cerchi di rendere conto, come auspicava già nel 1968 Bob Kennedy criticando il Pil, di "quello che rende la vita degna di essere vissuta". La cultura della tutela avrebbe in questa prospettiva un humus favorevole.

\section{LA MOBILITAZIONE DEI CITTADINI}

Internet ha creato grandi opportunità in termini di possibilità di accesso alla conoscenza e all'informazione. La messa a disposizione di contenuti anche di pregio ha permesso di rendere accessibile al pubblico materiali e documenti poco noti, di aumentare la visibilità delle collezioni e delle istituzioni, di facilitare la ricerca, di moltiplicare le possibilità di analisi e interpretazione dei contenuti, di meglio proteggere materiali o opere fragili, di creare le condizioni per la creazione di nuovi contenuti e di nuove possibilità di accesso. Questo ventaglio di possibilità tuttavia è spesso rimasto sulla carta; l'investimento in digitalizzazione non sempre ha portato ad un maggiore accesso ai contenuti, mentre in compenso diverse istituzioni hanno sperimentato la riduzione nel numero dei frequentatori fisici. Un esempio particolarmente rilevante è dato dalle biblioteche, pesantemente coinvolte in processi di digitalizzazione delle collezioni e di allargamento di offerta al pubblico (Darnton 2011), in un momento in cui il comportamento degli utenti e di parte dell'opinione pubblica, oltre ai tagli alle risorse disponibili, vanno nella direzione di una loro progressiva marginalizzazione (Palfrey 2015), portandole ad essere un riferimento fisico prevalente- 
mente per i gruppi sociali marginali, anzichè un catalizzatore di coesione sociale e culturale per le comunità in cui sono inserite.

Come fare dunque per approfittare delle opportunità della tecnologia per coinvolgere alla cultura della tutela la collettività tutta, così da poter generare attorno ad una istanza così importante un ampio consenso? Una tutela "militante" ha bisogno di uno spazio collettivo di dibattito (Boyte 2004 and 2007, Milner 2002, Putnam 2000) e di un coinvolgimento ampio che sia al tempo stesso partecipazione (Dzur 2008), corresponsabilizzazione e comportamento coerente individuale e collettivo (Boyte 2004). In questo modo, non solo il cittadino è più informato (Milner 2002), ma attraverso la partecipazione diventa anche più competente (Elkin, Soltan 1999). Se guardiamo l'evoluzione nel tempo del numero di votanti alla campagna FAI "I luoghi del cuore" non possiamo non rilevare che si tratta di un esperimento ben riuscito di mobilitazione su base locale. E' evidente che per poter essere il progetto vincitore, occorre che a livello locale si crei un fortissimo consenso e una fortissima attenzione al luogo da proteggere, il che crea le premesse non solo per accogliere l'intervento di restauro, ma la sua successiva presa in carico e valorizzazione da parte della collettività.

Il crowdsourcing - un modello di raccolta e condivisione di informazione che utilizza il web per mobilitare grandi numeri di persone per ottenere risultati significativi, specifici e condivisi (Howe 2006) - rappresenta un'altra modalità di mobilitazione di energie che ha permesso in ambiti diversi di raccogliere grandi volumi di informazioni in tempi brevi e a costi contenuti, facendo leva sulla expertise diffusa di professionisti (Ellis 2014), sull'intelligenza collettiva anche di non esperti (Surowieki 2004), motivati a collaborare (Lebraty, Lobre-Lebraty 2013). Sviluppato inizialmente in ambiti professionali o all'interno di grandi imprese localizzate in più paesi (Brabham 2008), il crowdsourcing è stato via via utilizzato in contesti diversi, al crescere dell'offerta di piattaforme digitali collaborative da un lato e dell'abitudine a condividere in rete materiali e informazioni dall'altro.

Diversi esempi testimoniano lo sforzo di istituzioni culturali a sviluppare progetti digitali partecipati che permettano al contempo coinvolgimento civico e qualità di risultato.

3 http://iluoghidelcuore.it/classifica/2014 
Nonostante vi siano diversi esperimenti in atto, le istituzioni legate al patrimonio culturale a livello internazionale sono state in genere molto prudenti a proporre progetti di questo tipo, per questioni di affidabilità e qualità dei risultati, difficoltà a mobilitare una massa critica di partecipanti e per una diffidenza da parte degli esperti legata al timore di vedere sminuite le proprie professionalità (Freedman 2000; Corsane 2005; Yang et al. 2014; Eveleigh 2014). Mentre il crowdsourcing è stato studiato con riferimento alla gestione dell'emergenza umanitaria in caso di conflitti e di disastri naturali (Yang et al. 2014) e applicato con successo in alcuni casi, come la gestione dell'emergenza al momento del terremoto ad $\mathrm{Haiti}^{4}$, i risultati più ambiziosi di progetti di crowdsourcing specificamente rivolti alla conservazione del patrimonio hanno finora riguardato le biblioteche; ne è un esempio il progetto di digitalizzazione di raccolte istituzionali e di raccolta degli archivi individuali realizzato a livello europeo in occasione del centenario della prima guerra mondiale o i progetti della New York Public Library, in grado contemporaneamente di far conoscere le raccolte digitali poco consultate, mobilitare la cittadinanza e creare nuova conoscenza in un processo collaborativo fra utilizzatori e personale della biblioteca (Autera 2016). Meno significativi sono stati i risultati di progetti crowdsourced di identificazione e catalogazione dei danni al patrimonio, come quello realizzato in occasione del terremoto in Nepal e coordinato dall'ICCROM ${ }^{6}$. Si tratta in ogni caso di esperimenti interessanti, sui quali lavorare per immaginare modalità collaborative di raccolta di informazioni, visualizzazione, analisi e supporto alle decisioni di intervento.

Le poche suggestioni che ho tracciato in questo intervento hanno l'obiettivo di mettere in luce come - benchè la tutela rimanga elemento distintivo delle politiche culturali nel nostro paese - sia necessario sviluppare una maggiore attenzione e un maggiore coinvolgimento attorno alla tutela da parte di una pluralità di attori, così da permettere la sua sostenibilità. Per quanto sia necessario rafforzare le organizzazioni pub-

4 Il resoconto di come la piattaforma è stata attivata e alimentata è disponibile qui http://voices.nationalgeographic.com/2012/07/02/crisis-mapping-haiti/

5 http://www.iccu.sbn.it/opencms/opencms/it/main/attivita/internaz/pagina_0001.html http://www.tapum.it/news/24-prima-guerra-mondiale-nasce-1-archiviodei-ricordi.html

6 https://kathmanduculturalemergency.crowdmap.com/main 
bliche responsabili della tutela del patrimonio, il loro operato deve inserirsi in una rete sociale che condivida e sostenga la loro azione. Il rinnovamento della cultura della tutela nella direzione di una mobilitazione collettiva, come suggerito da diversi autori, richiede di immaginare azioni ed interventi in più direzioni. La riforma del MIBACT in atto sta creando alcune condizioni istituzionali che favoriscono una maggiore collaborazione fra lo Stato e altri attori e che inevitabilmente si ripercuotono sui modi in cui esercitare l'azione di conservazione e di tutela. I poli museali e i musei autonomi avranno via via più bisogno e più incentivi a collaborare con gli altri attori chiave sul territorio, ma dovranno anche sviluppare e svilupperanno pratiche nuove nei rapporti con le sovrintendenze da una parte, le direzioni centrali dall'altra e gli attori pubblici e privati sul territorio. Alcune iniziative di successo ci dicono che è possibile immaginare e realizzare progetti anche di portata locale; ma lo spazio per l'innovazione è ancora molto ampio.

\section{BIBLIOGRAFIA}

Autera S., Digitalisation for citizens' involvement: New York Public Library in Dubini P. (a cura di) Institutionalising fragility, Milano, Fondazione Feltrinelli, 2016.

Baia Curioni S., L'intrapresa culturale. Riflessioni sul rapporto tra produzone culturale e logiche di impresa, Economia e Managemnet, 1(2006).

Bevan R., The destruction of Memory - Architecture at War, London, Reaktion Book, 2006.

Boyte H., Everyday Politics: Reconnecting Citizens and the Public Life, University of Pennsylvania Press, 2004.

Bruno I., La nascita del Ministero per i beni culturali e ambientali. Il dibattito sulla tutela, Milano, LED edizioni, 2011.

Cammelli M. (a cura di), Il Codice dei Beni Culturali e del Paesaggio, Bologna, Il Mulino, 2004.

Casini L. (a cura di), La globalizzazione dei Beni Culturali, Bologna, il Mulino, 2010 .

Casini L., Ereditare il futuro. Dilemmi sul patrimonio culturale, Bologna, il Mulino, 2016.

Cecchi R., I Beni culturali. Testimonianza materiale di civiltà, Milano, Spirali, 2006.

Cecchi R., Abecedario: come proteggere e valorizzare il patrimonio culturale italiano, Milano, Skira, 2015.

Chastel A., L'Italia, museo dei musei in Emiliani A. (a cura di) I musei Touring Club Italiano, 1980, 11-18.

Corsane G., Issues in Heritage, Museums and Galleries in Corsane G. (a cura di) Heritage, Museums and Galleries: An Introductory Reader. Abingdon UK; Routledge, 2005, 1-14. 
Darnton R., Six Reasons Google Books Failed, http://www.nybooks.com/daily/2011/ 03/28/six-reasons-google-books-failed/, 2011.

Della Torre S. e Petraroia P., Norme e pratiche senza sistema, Economia della cultura, 2, 2008, 161-172.

Della Torre S., Conservazione programmata: le implicazioni economiche di un cambio di paradigma, Il Capitale Culturale, 1, 2010, 47-55.

Dezzi Bardeschi M., Restauro: due punti e da capo (a cura di L. Gioeni), Milano, Franco Angeli, 2004.

Dzur A., Democratic Professionalism: Citizen Participation and the Reconstruction of Professional Ethics, Identity, and Practice, Pennsylvania State University Press, 2008.

Elkin S. and Soltan K., Citizen Competence and Democratic Institutions, Pennsylvania State University Press, 1999.

Ellis S., A bistory of collaboration, a future in crowdsourcing. Positive impacts of cooperation on British Librarianship, International Journal of Libraries and Information Services, 64:1, 2014, 1-10.

Eveleigh A., Crowdsourcing out the Archivist? Locating Crowdsourcing within the broader Landscape of Participatory Archives in Ridge M. (a cura di), Crowdsourcing our Cultural Heritage Surrey, Ashgate Publishing, 2014.

Forte P., La riforma organizzativa del MIBACT. I nuovi musei statali: un primo passo nella giusta direzione, Il Giornale delle Fondazioni, 15/09/2015.

Freedman G., The changing nature of museums, The museum journal, 43:4, 2000, 295 306.

Gasparoli P., La manutenzione preventiva e programmata del patrimonio storico tutelato come prima forma di valorizzazione, Techne 03/12, 2012, 148-157.

Howe J., Crowdsourcing: A Definition, http://crowdsourcing.typepad.com /cs/2006/06/crowdsourcing_a.html, 2006.

Istat, BES 2016 Il benessere equo e sostenibile, http://www.istat.it/it/files/2016/ 12/BES-2016.pdf, 2016.

Jalla D., La riforma dei musei statali italiani, il Giornale delle Fondazioni, 15/4/2015.

Jalla D., La sicurezza nei musei. Considerazioni e appunti introduttivi, Disponibile su http://www.academia.edu/10846307/La_sicurezza_nei_musei._Considerazioni _e_appunti_introduttivi_2015_.

Lebraty J.F., Lobre-Lebraty K., Crowdsourcing: a new form of externalisation. Crowdsourcng: one step beyond, Somerset NJ USA, John Wiley \& Sons, 2013.

Manacorda D., L'Italia agli italiani: istruzioni e ostruzioni per il patrimonio culturale, Bari, Edipuglia, 2014.

Milner H., Civic Literacy: How Informed Citizens Make Democracy Work, University Press of New England, 2002.

Montanari T., Istruzioni per l'uso del futuro, Roma, Minimum Fax, 2014.

Montella M., Valore e valorizzazione del patrimonio culturale storico, Milano, Electa, 2009.

Moreno Y.J., Santagata W., Tabassum A., Cultura materiale e sviluppo economico sostenibile, Sviluppo locale, XI, 26 (2/-2004-2005), 2004, 31-50. 
Musgrave R., The Theory of Public Finance: A Study in Public Economy, Mc Graw-Hill, 1959.

Palfrey J., Bibliotech: Why Libraries Matter More Than Ever in the Age of Google, New York, Basic Books, 2015.

Petraroia P., Tutela e valorizzazione in Montella M. Dragoni P. (a cura di), Musei e valorizzazione dei beni culturali, Bologna-Macerata, 2010.

Piergigli V., I "beni culturali": interpretazione evolutiva di una nozione giuridica consolidata in Piergigli V., Maccari A.L. (a cura di), Il Codice dei Beni Culturali e del Paesaggio tra teoria e prassi, Milano, Giuffré, 2006, 17-46.

Putnam R., Bowling Alone: The Collapse and Revival of American Community, New York, Simon and Schuster, 2000.

Sciullo G., Articolo 3 Tutela del patrimonio culturale in Cammelli M. (a cura di), Il Codice dei beni Culturali e del Paesaggio, Bologna, il Mulino, 2004.

Settis S., Battaglie senza eroi, Milano, Electa, 2005.

Slade T., Cultural Carnage (documentario), 2015.

Surowiecki J., The Wisdom of Crowds: Why the Many are Smarter than the Few and How Collective Wisdom Shapes Business, Economies, Societies, and Nations, New York, Doubleday, 2004.

Urbani G., Intorno al restauro a cura di B. Zanardi, Milano, Skira, 2000.

Yang D., Zhang D., Frank K., Robertson P., Jennings E., Roddy M., Lichtensern M., Providing real time assistance in disaster relief by leveraging crowdsourcing power, London, Springer, 2014. 